\title{
NASAL ADMINISTRATION OF RETINAL ANTIGENS MAINTAINS IMMUNOSUPPRESSION OF UVEORETINITIS IN CYCLOSPORIN-A-TREATED LEWIS RATS: FUTURE TREATMENT OF ENDOGENOUS POSTERIOR UVEORETINITIS?
}

\author{
BERNHARD KREUTZER, BARBARA LALIOTOU, Y. F. CHENG, JANET LIVERSIDGE, \\ JOHN V. FORRESTER and ANDREW D. DICK
}

Aberdeen

\begin{abstract}
SUMMARY
Purpose: Current treatment of autoimmune endogenous posterior uveoretinitis (EPU) is limited by drug toxicity, unpredictable relapses on dose reduction and resistance to therapy. Administration of autoantigens via gastrointestinal or respiratory mucosa prior to antigen exposure induces immune hyporesponsiveness (mucosal tolerance) to further antigen sensitisation. In this study we assessed whether mucosal tolerance induction was possible after immunisation with retinal antigens in experimental autoimmune uveoretinitis (EAU) in animals that were short-term immunosuppressed with cyclosporin A (CsA) to determine whether mucosal administration of retinal antigens can maintain immunosuppression in sensitised and immunosuppressed individuals.

Methods: Female Lewis rats were immunised with retinal extract (RE) and then treated as follows. Group 1 received no specific therapy and served as control; group 2 were fed CsA from day 7 to day 20 postimmunisation; group 3 received inhalational tolerance therapy with $\mathrm{RE}$ in addition to $\mathrm{CsA}$; tolerance therapy was continued after day 20 when CsA was stopped. Experiments varying the timing and dosage of both tolerising and immunising antigen were also performed, the details of which are described. Incidence, day of onset and clinical activity were recorded and histopathological assessment of intraocular inflammation, in particular the extent of autoimmune target-organ damage, was graded semiquantitatively.
\end{abstract}

From: Department of Ophthalmology, Medical School, University of Aberdeen, Scotland, UK.

Correspondence to: Dr Andrew Dick, Department of Ophthalmology, Medical School, University of Aberdeen, Foresterhill, Aberdeen AB9 2ZD, Scotland, UK. Tel: +44-1224-681818 ext 54093. Fax: +44-1224-685158. e-mail: opt034@abdn.ac.uk.
Results: Compared with controls and group 2, group 3 showed both a marked delay in disease onset and a reduction in disease severity. This effect was both dose and dose-timing dependent. Tissue damage assessed in terms of preservation of rod outer segments was significantly less in group 3 .

Conclusions: The success of combination therapy, clinically, remains unknown at present but these results support continuing present clinical trials of mucosal tolerance therapy and in particular have future implications for either maintaining or inducing immunosuppression in autoimmune diseases in combination with present immunosuppressive therapies.

In man, endogenous human posterior uveitis (EPU) is a sight-threatening, organ-specific autoimmune disease characterised by a chronic intraocular inflammation where leucocytic infiltration is seen in the choroid, retina and vitreous cavity, and from which one of the results is destruction of the photoreceptor cells of the retina. ${ }^{1,2}$ In common with other autoimmune conditions, present treatment of EPU combines systemic steroid therapy with low-dose cyclosporin A (CsA) and in severe cases additional cytotoxic agents such as azathioprine. ${ }^{3,4}$ Recently, newer immunotherapies, for example $\mathrm{FK}-506^{5}$ and monoclonal antibody (mAb) such as CAMPATH$1 \mathrm{H},{ }^{6}$ have been used with some success. However, immunosuppressive therapy is restricted by the need for long-term use, which gives rise to significant side effects and an undesirable incidence of disease relapse. Newer therapies which maintain a state of both immunological and disease-specific suppression are eagerly awaited. 
Putative retinal autoantigens in man, including retinal S-antigen (S-Ag), have been used to induce a model of $\mathrm{CD}^{+}$T-cell-mediated organ-specific inflammatory disease of the eye: experimental autoimmune uveoretinitis (EAU) ${ }^{7,8}$ This model parallels well the clinicopathological features of EPU $^{9}$ and has provided investigators with an excellent model for studying various alternative strategies of immunotherapy. ${ }^{10,11}$ For example, the first clinical use of CsA in uveitis arose from the knowledge that CsA could suppress EAU, whether given at the time of immunisation with $\mathrm{S}-\mathrm{Ag}$ or 7 days post-immunisation. ${ }^{12}$ Of great interest recently has been the immunosuppression of S-Ag-induced EAU by oral feeding of milligram quantities of $\mathrm{S}-\mathrm{Ag}$ or its immunodominant peptides, ${ }^{13,14}$ comparable to other models of autoimmune disease. ${ }^{15,16}$ Clinical trials of oral tolerance therapy in uveitis as well as multiple sclerosis and rheumatoid arthritis are in progress, ${ }^{17}$ although to date there is no conclusive evidence that oral tolerance therapy can suppress ongoing autoimmune disease.

Arguably a more physiological method of inducing immunological tolerance with lower doses of antigen is via administration of microgram doses of antigen via the nasorespiratory tract (inhalational tolerance induction) ${ }^{18}$ Using this approach we have shown that inhalational tolerance induction with retinal antigens can successfully suppress EAU. ${ }^{19,20}$ This form of inhalational tolerance is retinal-antigenspecific, and suppresses delayed type hypersensitivity reactivity, whilst maintaining $\mathrm{T}$-dependent antibody production. ${ }^{21}$ The true potential of this form of therapy, however, cannot be fully realised until at least two questions are resolved. Firstly, in all forms of tolerance induction for autoimmune disease it is still not known whether mucosal tolerance therapy suppresses active disease, although it has been shown experimentally that oral tolerance suppresses chronic relapsing $\mathrm{EAE}^{22}$ and passive ( $\mathrm{T}$ cell line) induction of disease. ${ }^{14}$ In the clinical setting it is of particular importance to establish whether this form of therapy would be effective in sensitised individuals with active disease, as there is a delicate balance between possible exacerbation of autoimmune disease and suppression when antigens are administered via mucosal lymphoid tissue. ${ }^{23}$ Secondly, the role of tolerance induction to maintain a state of immunoregulation in patients who respond favourably to steroids and CsA (i.e. are sensitised to autoantigen and immunosuppressed) is also not known. The latter is highly pertinent since many potential recruits to mucosal tolerance therapy will in the present clinical setting require or are at present requiring immunosuppressive therapy.

The aim of the paper was therefore to determine whether administration of retinal autoantigens via the nasorespiratory tract (inhalational tolerance) can suppress active EAU, either alone or in combination with oral CsA therapy, and maintain CsA-induced immunosuppression. Our approach was to evaluate the effect of oral CsA therapy combined with doses of retinal antigens administered intranasally (known to suppress EAU when given before immunisation), at various time intervals from 7 days after immunisation (i.e. after priming and generation of $\mathrm{CD}^{+} \mathrm{T}$ cells). Retinal antigens were administered as an unfractionated preparation of retinal extract (RE), which includes major uveitogenic retinal antigens, S$\mathrm{Ag}$ and interphotoreceptor retinoid binding protein (IRBP).

\section{Induction of EAU}

\section{METHODS}

Inbred adult female Lewis rats were obtained from the Animal Facilities, Medical School, University of Aberdeen. Six- to 8-week-old (150-200 g) rats were used in all experiments, which followed guidelines and approval of the Home Office, UK, for scientific experimentation and housing of animals. EAU was induced by intradermal footpad injections $(0.1 \mathrm{ml})$ with $5.7-6.7 \mathrm{mg} / \mathrm{ml}$ of $\mathrm{RE}$ w/v in complete Freund's adjuvant (CFA) containing $0.5 \mathrm{mg}$ H37RA $M$. tuberculosis. RE was prepared as previously described ${ }^{19,21}$ by hypotonic lysis of freshly dissected bovine retinae in the dark and contained uveitogenic proteins, including S-Ag and IRBP, as confirmed by SDS-PAGE electrophoresis (Pharmacia, Sweden) and western blot analysis.

\section{Inhalational Tolerance and Cs A Therapy}

We initially performed experiments with CsA alone at various time periods before and after immunisation, in order to establish a model where the kinetics of intraocular inflammation in RE-induced EAU could be modified so that any synergistic or additive effect on clinical response as well as histological disease of intranasal administration of retinal autoantigens with CsA therapy could be adequately assessed. Animals were fed $15 \mathrm{mg} / \mathrm{kg}$ per day of CsA v/v in olive oil (see below) for 7 days prior to immunisation with $5.7 \mathrm{mg} / \mathrm{ml}$ of RE w/v CFA, and for $0-7,7-13$ and 7-20 days following immunisation (Table I). Control animals were fed a weightmatched dose of carrier (olive oil).

The investigation of the effect of combined therapy with various doses of tolerising and immunising antigen over various time periods was performed where animals were treated with nasal administration of RE or gastric feeding of CsA or both. The protocol for timing of combined therapy was established from the experiment detailed above (see Results). The protein concentration of RE used in these experiments for both nasal administration 
and immunisation was $5.7-6.7 \mathrm{mg} / \mathrm{ml}$. The total nasal inoculum doses are detailed in the tables and the Results section. A dose of $15 \mathrm{mg} / \mathrm{kg}$ per day of CsA in $1: 1 \mathrm{v} / \mathrm{v}$ of olive oil (Boots, Nottingham, UK) was administered via gastric lavage using a 4 FG intravenous cannula (Portex, Hythe, Kent). RE was administered at $30 \mu \mathrm{l} /$ nostril per day by an Oxford micropipette as previously described. ${ }^{19.21}$ Animals were administered RE nasally, either alone or in combination with CsA therapy, for days 7-11 and 14-18 post-immunisation (i.e. a total of 10 inoculations) and in another group nasal RE inoculation was continued once weekly for a further 2 weeks (days 21 and 28 post-immunisation). The experiments were also repeated with higher nasal inoculating and immunising doses of RE in which nasal administration was also continued daily for days 7-35 postimmunisation. CsA in all these experiments was in the form of Sandimmune oral solution (Sandoz, Switzerland). Control animals received a weightmatched dose of olive oil via gastric lavage and/or $30 \mu \mathrm{l} /$ nostril of phosphate-buffered saline (PBS).

\section{Clinical and Histological Assessment of EAU}

Ocular examination was performed daily using slit lamp biomicroscopy. The day of onset, severity and course of uveitis were noted. Inflammation was graded 0-4 according to the degree of cellular/ inflammatory activity within the anterior chamber of the eye. ${ }^{19}$ Histological examination was performed at the end of the experiment (days 21-35). Experimental animals were killed by $\mathrm{CO}_{2}$ asphyxiation and both eyes removed immediately and immersed in $2.5 \%$ glutaraldehyde in $0.1 \mathrm{M}$ phosphate buffer, $\mathrm{pH}$ 7.2. Semi-thin sections were obtained to incorporate the entire uvea including sclera, choroid, retina, ciliary body, lens, iris and cornea. Tissue was processed in the conventional manner and stained routinely with haematoxylin and eosin. At least three sections from each eye were examined for histological scoring under conventional bright field microscopy. Histological scores were obtained using a semiquantitative scoring system previously described. $^{20}$ Scores were statistically evaluated using the Student's $t$-test and $p<0.05$ was considered significant.

\section{Delayed Hypersensitivity Reactivity}

Skin tests were performed on day 14 post-immunisation. First the animal's flanks were shaved and intradermal injections of $0.1 \mathrm{ml}$ PBS, purified protein derivative (PPD; $100 \mu \mathrm{g})$ and RE $(100 \mu \mathrm{l} ; 5.7 \mathrm{mg} / \mathrm{ml}$ $\mathrm{RE}$ ) were given in separate sites in the flank. The injection sites were noted for erythema and induration, and the increase in skin thickness was measured with a micrometer gauge at $4,12,24$ and 48 hours post-injection. The extent of the swelling was calculated by subtracting the value for the PBSinjected site from that of the test sites (PPD and RE).

\section{Cs A Therapy in Active EAU}

Prior to assessing combined mucosal tolerance and CsA therapy we established a model of CsAmodified EAU, whereby at varying time points administration of CsA could modify the course of EAU, in particular with respect to both day of onset and clinical severity of intraocular inflammation (Table I). Clinically, intraocular inflammation was completely abrogated when CsA was administered at $15 \mathrm{mg} / \mathrm{kg}$ per day during priming of the immune response, i.e. from day 0 to 7 post-immunisation with RE. Pretreatment with CsA before immunisation had no protective effect when compared with controls (Table I). Of note is that when administering CsA after priming (from day 7 post-immunisation onward) its effectiveness was mostly a delay in the onset of disease with only a minimal reduction in intraocular inflammation clinically. This effect was dependent upon the length of time CsA was continued. Onset of disease in animals treated from days 7 to 13 post-immunisation occurred on day 18 compared with day 29 in animals treated from days 7 to 20 post-immunisation, and day 12 in control animals (Table I). There was no significant difference in mean maximal clinical severity between the groups (mean scores of 2.8, 3 and 4, respectively). In all animals treated with CsA after immunisation there was a significant reduction in delayed hypersensitivity reactivity (DTH) to RE which was not apparent in animals treated with CsA before immunisation (data not shown), confirming a suppression of $\mathrm{Th} 1 \mathrm{CD}^{+} \mathrm{T}$ cell reactivity with CsA treatment.

Therefore, to assess any possible effect of combined therapy of CsA with nasal tolerance induction we chose to combine therapies after immunological priming from day 7 post-immunisation onward, where CsA therapy alone only modifies onset of disease and not severity. Firstly, this allows an

Table I. CsA therapy in experimental autoimmune uveoretinitis

\begin{tabular}{cccc}
\hline $\begin{array}{c}\text { CsA therapy } \\
\text { (days pre- } \\
\text { or post- } \\
\text { immunisation) }\end{array}$ & $\begin{array}{c}\text { Incidence } \\
\text { (animals) }\end{array}$ & $\begin{array}{c}\text { Day of } \\
\text { onset }\end{array}$ & $\begin{array}{c}\text { Maximal } \\
\text { mean } \\
\text { clinical } \\
\text { severity }\end{array}$ \\
\hline $\begin{array}{c}\text { Nil } \\
-7 \text { to } 0\end{array}$ & $8 / 8$ & 12 & 4 \\
0 to 7 & $6 / 6$ & 12 & 4 \\
7 to 13 & $0 / 8$ & -18 & 0 \\
7 to 20 & $6 / 8$ & 18 & 2.8 \\
\hline
\end{tabular}

Cyclosporin A (CsA) was administered as $15 \mathrm{mg} / \mathrm{kg}$ per day via gastric lavage $v / v$ in olive oil and animals were immunised with retinal extract (RE) in complete Freund's adjuvant (CFA). Histology grading was performed from days 21 or 35 postimmunisation, i.e. at the end of experiment for each group, with resolution of clinical disease. 
Table II. Clinical and histology scores of EAU modulated by nasal administration of RE in CsA-treated animals

\begin{tabular}{cccccc}
\hline & $\begin{array}{c}\text { Nasal } \\
\text { administration } \\
\text { of antigen }\end{array}$ & $\begin{array}{c}\text { Incidence } \\
\text { (animals) }\end{array}$ & Day of onset & \multicolumn{2}{c}{ Clinical } \\
\cline { 3 - 6 } Nil & Nil & $4 / 4$ & 10 & $3.8 \pm 0.5$ & $3.5 \pm 0.4$ \\
Nil & $7-20$ & $4 / 4$ & 13 & $4.4 \pm 0.25$ & $3.2 \pm 0.8$ \\
$7-20$ & Nil & $4 / 4$ & 25 & $3.8 \pm 0.5$ & $3.5 \pm 0.3$ \\
$7-20$ & $7-20$ & $4 / 6$ & 29 & $2.0 \pm 0$ & $2.4 \pm 0.6^{*}$ \\
$7-20$ & $7-35$ & $4 / 4$ & 28 & $2.6 \pm 0.8$ & $2.3 \pm 0.5^{*}$ \\
\hline
\end{tabular}

*There was significant $(p<0.01)$ suppression of histological disease in animals treated with both CsA and nasal administration of retinal antigen compared with controls and animals treated with CsA only. Score is derived from an average of affected eyes in each group. All therapy described in the table commenced after immunisation with RE in CFA. These results are representative data of duplicate experiments. '7-20' refers to nasal administration of antigens or CsA feeding which was performed from days 7-11 and 14-18 postimmunisation. In one group nasal antigen administration was continued once weekly until day 35 (days 21 and 28).

assessment of any further delay in onset of EAU which might occur and secondly to what extent suppression of intraocular inflammation could be induced and maintained when additional nasal administration of retinal antigens was given (inhalational tolerance). This experimental scenario has the added value of more accurately representing the clinical setting where many patients are already treated with CsA.

\section{Nasal Administration of Antigens in Combination with CsA Therapy in Active EAU}

We have previously shown that inhalational tolerance induction with microgram doses of retinal antigens gives rise to an antigen-specific suppression of DTH reactivity and clinical and pathological disease suppression when administered prior to immunisation. ${ }^{19,21}$ Using a similar dose regimen after immunisation, we attempted to modulate EAU during both immune priming and active disease with or without combination therapy with oral CsA. Given that CsA therapy (see above) provides a delay in onset of disease, but the incidence and severity of disease remain unchanged when given from day 7 to day 20 post-immunisation, assessment of whether combined therapy maintains or further suppresses EAU can be made. Table II shows that animals receiving tolerogenic doses of antigen via the nasorespiratory tract (total dose received of retinal extract is $3.4 \mathrm{mg}^{19}$ over a period from day 7 to day 20 (days 7-11, 14-18) postimmunisation with $250 \mu \mathrm{g}$ of $\mathrm{RE}$ were unable to suppress clinical and histological disease in EAU (mean histological scores of $3.5 \pm 0.4$ controls; $3.2 \pm$ 0.8 nasal RE group). However, when combined nasal administration of RE and oral CsA therapy was given for the same time period (over a period of day 7 to day 20 post-immunisation), the onset of disease was delayed. Furthermore, both clinical and histological severity were significantly suppressed $(2.0 \pm 0$, $2.4 \pm 0.6$ respectively, compared with controls $3.8 \pm$ $0.5,3.5 \pm 0.4$; Table II). As we have previously shown (see above) CsA therapy alone delayed onset of disease without suppressing either the clinical or histological inflammatory response (clinical severity of $3.8 \pm 0.5$; histology score $3.5 \pm 0.3$; Table II). When nasal administration of RE was continued weekly after CsA therapy had stopped, the major effect was not a significant further delay in onset of disease but maintained suppression of clinical and histological disease (histology scores of $2.3 \pm 0.5$ in combined therapy and $3.5 \pm 0.3$ in CsA therapy alone, $p<0.01$; clinical scores of $2.6 \pm 0.8$ in combined therapy and $3.8 \pm 0.5$ in CsA therapy alone, $p<0.05$; Table II). These results are representative of repeated independent experiments where in all experiments there was a significant delay in onset of disease compared with controls when combined therapy was given. Furthermore, in one experiment all three animals in the combined treatment group failed to develop any signs of disease (either clinically or histologically) when inhalational tolerance was continued weekly, following CsA therapy, up to day 45 post-immunisation.

Fig. 1 shows the histological findings at day 35 post-immunisation in the various treatment groups, and demonstrates the protective effect of combined CsA and inhalational tolerance therapy. In control, untreated animals there was marked retinal destruction with loss of target-organ rod outer segments (ROS loss; Fig. 1B, arrow) which was similar to the destruction of ROS in animals treated with CsA only (Fig. 1C, arrowhead), despite a delay in onset of disease. Note at this stage there is still considerable retinal infiltrate via the inner retinal vessels (Fig. 1C, arrow). A combination of CsA therapy with inhalational tolerance, however, demonstrates a marked reduction in ROS loss. Only focal mononuclear retinal cell infiltrate is apparent (Fig. 1D, arrow), and the majority of ROS remain unaffected (Fig. $1 \mathrm{D} ; \mathrm{R})$.

We further assessed whether higher doses of intranasal RE when administered daily throughout the treatment period offered any additional effect. In these experiments, protein concentration of RE used was $6.7 \mathrm{mg} / \mathrm{ml}$. RE was administered daily for days $7-20$ or $7-35$ post-immunisation $(0.1 \mathrm{ml})$ with $350 \mu \mathrm{g}$ of RE (a total inoculum dose of $5.3 \mathrm{mg}$ and $11.3 \mathrm{mg}$ of protein respectively) with combined oral CsA therapy as described before. The results show that 

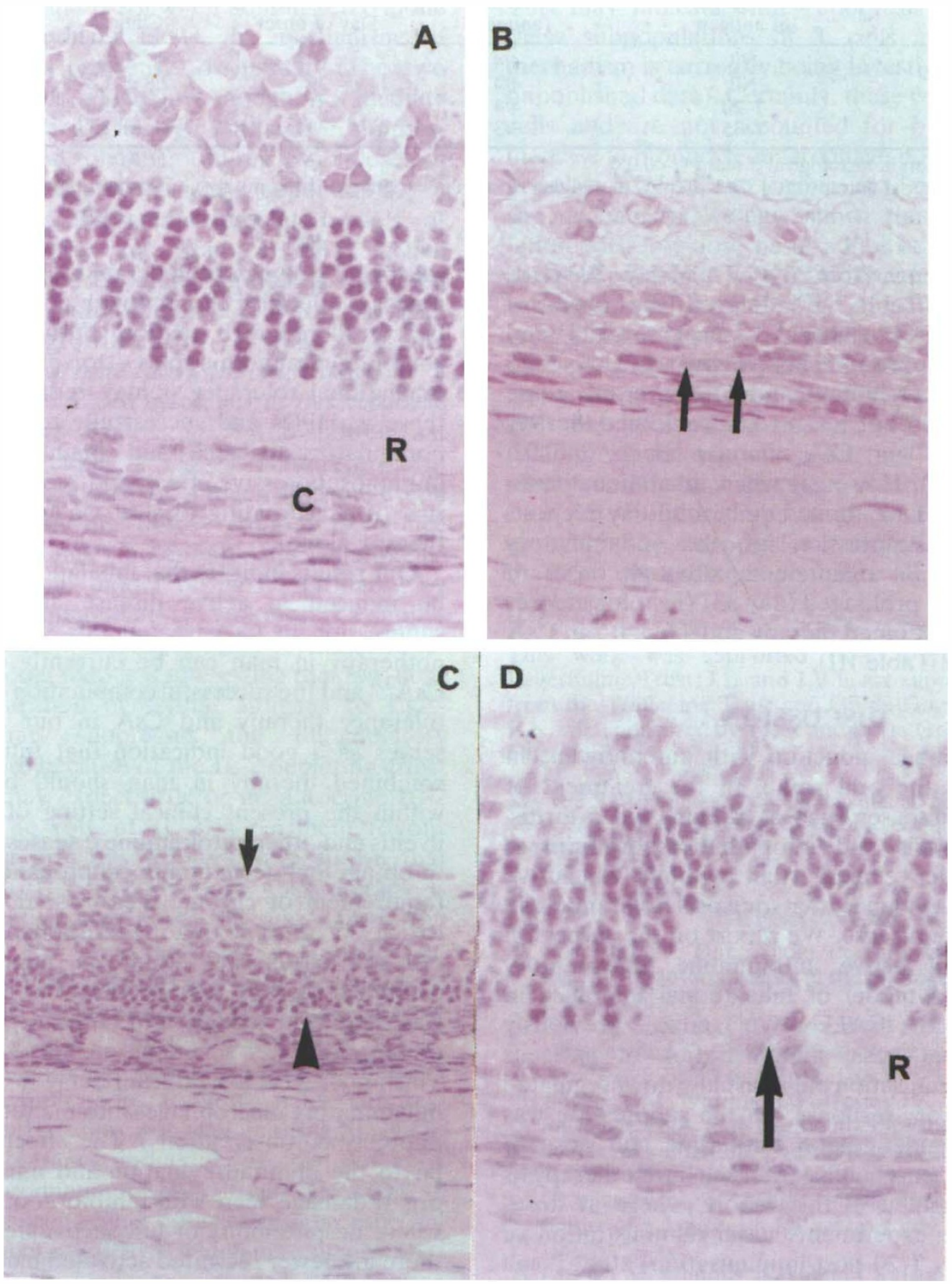

Fig. 1. Histological features of experimental autoimmune uveitis (EAU) in animals treated with cyclosporin $A(C s A)$ with or without inhalational tolerance therapy and in control animals. $(A)$ The normal anatomy of a Lewis rat retina, where the rod photoreceptors $(R)$ are intact. $C$, choroid. $\times$ 400. (B) Animals immunised with RE/CFA demonstrate the classical features of experimental autoimmune uveoretinitis. There is total target-organ destruction manifested by the complete loss of rod photoreceptor outer segments (ROS; arrow). $\times 400$. (C) In animals treated with CsA alone (i.e. from days 7 to 20 post-immunisation with $250 \mu \mathrm{g}$ of $R E$ ) there is still considerable ROS loss (arrowhead) and inner retinal vasculitis (arrow). $\times 250$. (D) Combined therapy including intranasal administration of retinal antigens ( $R E$ at $5.7 \mathrm{mg} / \mathrm{ml}$ ) from days 7 to 12 and 15 to 20 post-immunisation results in protection of ROS $\times 400$, where only focal infiltration of the retina was seen (arrow). 
Table III. Higher doses of RE administered daily to CsA-treated animals abrogates clinical and histological suppression of EAU

\begin{tabular}{|c|c|c|c|c|c|}
\hline \multirow[b]{2}{*}{ CsA therapy } & \multirow{2}{*}{$\begin{array}{c}\text { Nasal } \\
\text { administration } \\
\text { of antigen }\end{array}$} & \multirow{2}{*}{$\begin{array}{l}\text { Incidence } \\
\text { (animals) }\end{array}$} & \multirow[b]{2}{*}{ Day of onset } & \multicolumn{2}{|c|}{ Mean maximal severity $( \pm S D)$} \\
\hline & & & & Clinical & Histology \\
\hline Nil & Nil & $4 / 4$ & 10 & $4.2 \pm 0$ & $4.2 \pm 0.5$ \\
\hline $7-20$ & Nil & $4 / 4$ & 29 & $2.8 \pm 1.8$ & $4.6 \pm 0.7$ \\
\hline $7-20$ & $7-20$ & $4 / 4$ & 31 & $1.4 \pm 1.8$ & $1.8 \pm 1.2 *$ \\
\hline $7-20$ & $7-35$ & $3 / 3$ & 33 & $3 \pm 1.1$ & $4 \pm 1.1^{* *}$ \\
\hline
\end{tabular}

*Significant reduction in histological disease $(p<0.05)$.

**Continuing intranasal therapy after CsA therapy at a higher dose than experiment 1 did not result in any protection despite a delay in disease onset.

combined therapy from day 7 to day 20 postimmunisation (Table III) delayed the onset of disease (day 31), reduced clinical severity and significantly suppressed histological disease compared with no treatment and CsA therapy alone (histological scores of $1.8 \pm 1.2$ in combined therapy and $4.6 \pm 0.7$ in CsA therapy alone; $p<0.02$, Student's $t$-test). However, when inhalational therapy with RE was continued daily until day 35 postimmunisation suppression of the inflammatory response was not maintained, although onset of disease was still prolonged (day 33) (histology scores of $4 \pm 1.1$ in combined therapy and $4.6 \pm 0.7$ in CsA therapy alone; Table III).

\section{DISCUSSION}

Mucosal tolerance induction with autoantigens, in particular via the oral route, in the treatment of autoimmune diseases such as rheumatoid arthritis, multiple sclerosis and idiopathic uveoretinitis is currently under clinical trial, without conclusive experimental evidence that mucosal tolerance can suppress active disease. We report on the successful suppression of active autoimmune inflammatory responses in a model of inhalational tolerance in CsA-modulated EAU. We have previously reported $^{20}$ that nasal administration of antigens following immunisation only partially downregulated the inflammatory response but the suppression was variable, dependent upon both time and dose of therapy. The unreliability of such monotherapy is further highlighted in this report, where at doses used in these experiments nasal administration of antigens (days 7-20 post-immunisation) after $\mathrm{T}$ cell priming and before clinical disease was unsuccessful in suppressing active EAU, despite a slight delay in disease onset. The fact that nasal administration of $\mathrm{RE}$ does generate suppression is encouraging and helps, in part, to support the continuance of present clinical trials with mucosal tolerance therapy. ${ }^{17}$ However, the variable response and strict dependence upon timing of treatment and dose of antigen given, highlights the potential difficulties which may occur in securing suppression clinically. Furthermore, the dose, timing and route of administration of tolerising antigen is particularly important as experimentally both regulatory $\mathrm{T}$ cells as well as auto- reactive T cells can be generated. ${ }^{23}$ Therefore clinical mucosal tolerance therapy with autoantigens has to be considered with caution. Future therapies that combine immunotherapies - for example, CsA and inhalational tolerance - may reduce the effect of these variables and successfully redress the immunoregulatory balance and maintain the desired immunosuppressive effect, and reduce the potential risk of exacerbating disease via mucosal tolerance therapy alone.

Our results indicate that inhalational tolerance can be induced in active disease and can maintain suppression in CsA-treated rats. Successful immunotherapy in man can be currently obtained with $\mathrm{CsA}^{3,4}$ and the successful combination of inhalational tolerance therapy and CsA in our animal model serves as a good indication that future studies of combined therapy in man should be undertaken within the present clinical setting of treatment of uveitis and other autoimmune diseases, in an attempt to obtain long-term immunosuppression without the requirement of chronic therapy and to reduce the toxicity of present therapy. Although inflammatory cell infiltration was found in animals receiving combined therapy, it is important to note that there was significant reduction in target-organ destruction of the rod outer segments on histological grading. This may reflect a significant delay in recruitment of inflammatory macrophages into the retina (Kreutzer, unpublished observations). The effect appears similar to the immunoregulation and minimised targetorgan damage seen with inhibition of TNF activity, where despite influx of fully activated $\mathrm{CD}^{+} \mathrm{T}$ cells there are fewer recruited activated macrophages and granulocytes in retinae. ${ }^{24}$ The effect of combined therapy on cytokine production and the anti-inflammatory role of cytokines in this model are currently under investigation.

We are presently investigating the possible mechanisms of this synergy. Our results show that combined therapy is again dependent upon dose and timing of nasal administration of retinal antigens, as increasing dose and frequency can abrogate this synergistic response (Table III), where daily nasal administration of RE continuing after cessation of CsA therapy results in marked ROS destruction. The suppressive effect of administering RE intranasally 
prior to immunisation is mainly accounted for by IRBP and S-Ag and the effect is antigen-specific. ${ }^{25}$ Also both IRBP and S-Ag when administered alone can inhibit RE-induced EAU, the mechanisms of which have been previously discussed. ${ }^{25}$ The two major mechanisms which are thought to mediate mucosal tolerance are anergy and active suppression. ${ }^{26}$ Inhalational tolerance utilises lower doses of antigen and is mediated by active suppression. ${ }^{21.27}$ Furthermore, in other experimental models of autoimmune disease, for example EAE, active suppression induced by oral tolerance is regulated by cells secreting anti-inflammatory cytokines, such as transforming growth factor (TGF- $\beta$ ). ${ }^{28}$ As the suppression of low zone tolerance is mediated by $\mathrm{T}$ cells, ${ }^{26}$ the role of $\mathrm{T}$ cell cytokines in this response was investigated by Rizzo et al. ${ }^{29}$ who showed that exogenous interleukin 2 (IL-2) administered in animals fed lower doses of antigen (although still much higher than the doses required to induce inhalational tolerance) potentiated the tolerogenic effect in a murine model of EAU, probably by secretion of anti-inflammatory cytokines. If a similar active suppressive mechanism exists for nasal tolerance in this model then it is interesting that low-dose nasal administration of retinal antigens to CsAtreated animals suppresses the inflammatory response, where because of the IL-2 depleting effect of CsA, one may expect little or no autocrine IL-2 production. The role of IL-2 is pertinent to our model, in that previous workers have shown that combined anti-IL-2 and low-dose CsA therapy is unable to inhibit active EAU but does inhibit the uveitogenic $\mathrm{T}$ cell transfer model of EAU. ${ }^{30}$ The levels and time response of IL-2 production and IL-2 secreting $\mathrm{T}$ cells are important for the efficacy of such treatment. However, in our model the levels of IL-2 may have been sufficient locally (respiratory mucosa and drainage lymph nodes) to induce active suppression, or indeed IL-2 may not play an integral role in inhalational tolerance induction at low doses or generate the production of IL-4 driven, $\mathrm{Th} 2 \mathrm{CD}^{+} \mathrm{T}$ cells. When we used higher doses of antigen, mucosal tolerance induction was lost, which may suggest that at this dose either autoreactive $\mathrm{T}$ cells were generated ${ }^{23}$ or tolerance induction is IL-2 dependent. The former is less likely as generation of autoreactive $\mathrm{T}$ cells requires IL-2 for proliferation and activation and would be expected to be absent in the presence of CsA. Recent evidence suggests that $\gamma \delta \mathrm{T}$ cells mediate active suppression in inhalational tolerance, ${ }^{31,32}$ although in our model of administering antigens nasally prior to immunisation, we have no direct support for this mechanism. We have shown that tolerised animals (pre-immunisation) that are subsequently protected against RE-induced disease have a population in the nasorespiratory drainage lymph nodes of both $\mathrm{CD}^{+}$and $\mathrm{CD} 8^{+} \mathrm{T}$ cells which have down-regulated $\alpha \beta \mathrm{T}$ cell receptor expression. This may indicate either apoptosis or deletion of these subpopulations of $\mathrm{T}$ cells and the precise mechanism is currently being investigated (Laliotou, unpublished data). Certainly, these cells are not $\gamma \delta \mathrm{T}$ cells and are not accounted for by a concurrent increase in monocyte/macrophage population or by a population of recent thymic immigrants. Whatever the mechanisms, we have shown that immunomodulation with low-dose nasal administration of retinal antigens combined with CsA is successful not only in suppressing inflammation but in maintaining longerterm immunoregulation and minimising target-organ damage. Pharmacological manipulation of immune responses (e.g. with CsA) will yield further insights into the cellular mechanisms and cytokine production in mucosal tolerance induction. In turn, this will identify both the dose range and timing of therapy required to induce suppression and not potentially abrogate any suppression induced by concurrent therapy or at worst enhance the autoimmune response.

This work was supported from a grant from the Leverhulme Trust. J.L. and J.V.F. are supported by grants from the Wellcome Trust and Guide Dogs for the Blind. B.K. was sponsored by Ernst and Berta Grimmke Stiftung, Dusseldorf.

Key words: Autoimmunity, Experimental autoimmune uveoretinitis (EAU), Cyclosporin A (CsA), Mucosal tolerance, Immunosuppression, Uveitis.

\section{REFERENCES}

1. Forrester JV. Duke Elder Lecture. New concepts on the role of autoimmunity in the pathogenesis of uveitis. Eye 1992;6:433-7.

2. Gery I, Mochizuki M, Nussenblatt RB. Retinal specific antigens and the immunopathologic mechanisms they provoke. Prog Retinal Res 1986;5:75-109.

3. Towler HM, Whiting P, Forrester JV. Combination of low dose CsA and steroid therapy of chronic intraocular inflammation. Eye 1990;4:514-20.

4. Towler HM, Forrester JV, Lightman SL. Low dose CsA therapy of ocular inflammation: preliminary report of a long term follow up study. Autoimmunity 1992;5:259-64.

5. Ishioka M, Ohno S, Nakamura S, Isobe K, Watanabe N, Ishigatsubo Y, Tanaka S. FK 506 treatment of noninfectious uveitis. Am J Ophthalmol 1994;118:723-30.

6. Isaacs J, Dick AD, Haynes RH, Watson P, Meyer PAR, Forrester JV, et al. Monoclonal antibody therapy of chronic intraocular inflammation using CAMPATH1H. Br J Ophthalmol 1995;79:1054-5.

7. Caspi RR, Roberge FG, McAllister CG, El-Saied M, Kuwabara T, Gery I, Hanna E, Nussenblatt RB. T cell lines mediating experimental autoimmune uveitis in the rat. J Immunol 1986;136:928-33.

8. Dick AD. Retinal antigen-specific $T$ cells mediate experimental autoimmune uveoretinitis (EAU) in PVG rat: a model for tracking antigen-specific $\mathrm{CD}^{+}$ $\mathrm{T}$ cells in the inflamed eye. Ocul Immunol Inflam 1995;3:261-70. 
9. Forrester JV, Liversidge J, Dua HS, Towler H, McMenamin PG. Comparison of clinical and experimental uveitis. Curr Eye Res 1990;9:75-84.

10. Dick AD. Experimental approaches to specific immunotherapies in autoimmune disease: future treatment of endogenous posterior uveitis. $\mathrm{Br} \mathrm{J}$ Ophthalmol 1995;79:81-8.

11. Forrester JV, Liversidge J, Dua HS, Dick A, Harper F, McMenamin PG. Experimental autoimmune uveoretinitis: a model system for immunosuppression: a review. Curr Eye Res 1992;11:33-42.

12. Nussenblatt RB, Rodrigues MM, Wacker WB, Cevario SJ, Salinas-Camara MC, Gery I. Cyclosporin A: inhibition of experimental autoimmune uveitis in Lewis rat. J Clin Invest 1981;67:1228-332.

13. Nussenblatt RB, Caspi RR, Mahdi R, Chan CC, Roberge F, Lider O, Weiner HL. Inhibition of S-Ag induced experimental autoimmune uveoretinitis by oral induction of tolerance with S-antigen. J Immunol 1990,144:1689-95.

14. Vrabec TR, Gregerson DS, Dua HS, Donoso LA. Inhibition of experimental autoimmune uveoretinitis by oral induction of tolerance with $\mathrm{S}-\mathrm{Ag}$ and synthetic peptides. Autoimmunity 1992;12:175-84.

15. Lider O, Santos LMB, Lee CYC, Higgens PJ, Weiner HL. Suppression of EAE by oral administration of myelin basic protein: suppression of the disease in vitro immune responses is mediated by antigen-specific $\mathrm{CD}^{+}$cells. J Immunol 1988;142:748-53.

16. Thompson HSG, Harper N, Bevan DJ, Staines NA. Suppression of collagen induced arthritis by oral administration of type II collagen: changes in immune and arthritis responses mediated by active peripheral suppression. Autoimmunity 1993;16:189-99.

17. Weiner HL, Mackin GA, Matsui M, Orav EJ, Khoury SJ, Dawson DM, Haffler DA. Double blind pilot trial of oral tolerisation with myelin antigens in multiple sclerosis (see comments). Science 1993;259:1321-4.

18. Holt PG, Sedgwick JD. Suppression of IgE responses following inhalation of antigens. Immunol Today 1987;8:14-5.

19. Dick AD, Cheng Y, Liversidge J, McKinnon A, Forrester JV. Nasal administration of retinal antigens suppresses the inflammatory response in experimental autoimmune uveoretinitis: a preliminary report of intranasal induction of tolerance with retinal antigens. Br J Ophthalmol 1993;77:171-9.

20. Dick AD, Cheng YF, Liversidge J, Forrester JV. Immunomodulation of experimental autoimmune uveoretinitis: a model of tolerance induction with retinal antigens. Eye 1994;8:52-9.

21. Dick AD, Cheng YF, Liversidge J, Forrester JV. Intranasal administration of retinal antigens suppresses retinal antigen induced experimental autoimmune uveoretinitis. Immunology 1994;82:625-31.
22. Kennedy MK, Tan LJ, DalCanto MC, Tuohy VK, Lu A, Trotter JL, Miller SD. Inhibition of murine relapsing EAE by immune tolerance to proteolipid protein and its encephalogenic peptides. J Immunol 1990;145:909-15.

23. Chen Y, Imobe I, Kichroo VK, Baron JL, Janeway CA $\mathrm{Jr}$, Weiner HL. Oral tolerance in MBP T cell receptor transgenic mice: suppression of autoimmune encephalomyelitis and dose dependent induction of regulatory cells. Proc Natl Acad Sci USA 1996;93:338-91.

24. Dick AD, McMenamin PG, Korner H, Scallon BJ, Ghrayeb T, Forrester JV, Sedgwick JD. Inhibition of tumour necrosis factor-activity minimises target-organ damage in experimental autoimmune uveoretinitis despite quantitatively normal activated $\mathrm{T}$ cell traffic to the retina. Eur J Immunol 1996;26:1018-25.

25. Laliotou B, Liversidge J, Forrester JV, Dick AD. Interphotoreceptor retinoid binding protein (IRBP) is a potent tolerogen in Lewis rat: suppression of experimental autoimmune uveoretinitis (EAU) is retinal antigen specific. Br J Ophthalmol 1997; 81:61-7.

26. Gregerson DS, Obritsch WF, Donoso LA. Oral tolerance in experimental autoimmune uveoretinitis: distinct mechanisms of resistance are induced by low dose vs high dose feeding protocols. J Immunol 1993;151:5751-61.

27. Sedgwick JD, Holt PG. Induction of IgE-secreting cells and IgE isotype specific suppressor cells in the respiratory tract lymph nodes of rats in response to antigen inhalation. Cell Immunol 1985;94:182-90.

28. Khoury SJ, Hancock WW, Weiner HL. Oral tolerance to myelin basic protein and natural recovery from experimental autoimmune encephalomyelitis are associated with downregulation of inflammatory cytokines and differential upregulation of transforming growth factor, interleukin 4, and prostaglandin E expression in the brain. J Exp Med 1992;176:1355-64.

29. Rizzo LV, Miller-Rivero NE, Chan CC, Wiggert B, Nussenblatt RB, Caspi RR. Interleukin-2 treatment potentiates induction of oral tolerance in a murine model of autoimmunity. J Clin Invest 1994;94:1668-72.

30. Higuchi M, Diamantstein T, Osawa H, Caspi RR. Combined anti-interleukin 2 receptor and low dose cyclosporine therapy in experimental autoimmune uveoretinitis. J Autoimmun 1991;4(1):113-24.

31. McMenamin C, Primm C, McKersey M, Holt PG. Regulation of IgE responses to inhaled antigens in mice by antigen-specific gamma-delta $\mathrm{T}$ cells. Science 1994;265:1869-70.

32. Wildner G, Kuhnlein P, Hunig T, Thurau SR. The role of gamma-delta $\mathrm{T}$ cells in induction and suppression of experimental autoimmune uveitis. Presented at the Joint European Research Meetings in Ophthalmology and Vision, Montpellier, France, October 1995. Abstract published in Vision Res 1995;35(Suppl):S113. 